\title{
Efficient high-throughput SARS-CoV-2 testing to detect asymptomatic carriers
}

\author{
Noam Shental ${ }^{1, *}$, Shlomia Levy ${ }^{2,3}+$, Vered Wuvshet ${ }^{2,3}+$, Shosh Skorniakov ${ }^{2,3}+$, Bar Shalem $^{4}$, Aner Ottolenghi ${ }^{2,3}$, \\ Yariv Greenshpan ${ }^{2,3}$, Rachel Steinberg ${ }^{5}$, Avishay Edri ${ }^{2,3}$, Roni Gillis ${ }^{6}$, Michal Goldhirsh $^{6}$, Khen Moscovici ${ }^{6}$, Sinai \\ Sachren $^{3}$, Lilach M. Friedman ${ }^{2,3}$, Lior Nesher ${ }^{5}$, Yonat Shemer-Avni' ${ }^{2,5}$, Angel Porgador ${ }^{2,3^{*}}$, Tomer Hertz ${ }^{2,3,7^{*}}$
}

${ }^{1}$ Department of Computer Science, The Open University of Israel. ${ }^{2}$ Department of Microbiology and Immunology, Ben-Gurion University of the Negev, Israel. ${ }^{3} \mathrm{National}$ Institute of Biotechnology in the Negev, Ben-Gurion University of the Negev, Israel. ${ }^{4}$ Department of Computer Science, Bar-Ilan University, Israel. ${ }^{5}$ Soroka University Medical Center, Beer-Sheva, Israel. ${ }^{6}$ Goldman Medical School, Faculty of Health Sciences, Ben Gurion University of the Negev, Israel. ${ }^{7}$ Vaccine and Infectious Disease Division, Fred Hutch Cancer Research Center

$\dagger$ These authors contributed equally to this work

*Co-senior author and co-corresponding author. Email: angel@bgu.ac.il (A.P.); shental@openu.ac.il (N. S.); thertz@bgu.ac.il (T. H.)

Recent reports suggest that $10-30 \%$ of SARS-CoV-2 infected patients are asymptomatic and that significant viral shedding may occur prior to symptom onset. Therefore, there is an urgent need to increase diagnostic testing capabilities to prevent disease spread. We developed P-BEST - a method for PoolingBased Efficient SARS-CoV-2 Iesting which identifies all positive subjects within a large set of samples using a single round of testing. Each sample is assigned into multiple pools using a combinatorial pooling strategy based on compressed sensing designed for maximizing carrier detection. In our current study we pooled sets of 384 samples into 48 pools providing both an 8 -fold increase in testing efficiency, as well as an 8-fold reduction in test costs. We successfully identified up to 5 positive carriers within sets of 384 samples. We then used P-BEST to screen 1115 healthcare workers using 144 tests. P-BEST provides an efficient and easy-to-implement solution for increasing testing capacity that can be easily integrated into diagnostic laboratories.

\section{Introduction}

The COVID-19 pandemic is rapidly spreading throughout the world. Recent reports suggest that $10-30 \%$ of SARS-CoV-2 infected patients are asymptomatic (1-5). Other studies report that some subjects have significant viral shedding prior to symptom onset (6). There have been multiple recent studies that identified asymptomatic carriers and have attempted to estimate their rate and contribution to disease spread. A study of 2685 tourists in the New York area conducted over two seasons found that $6.2 \%$ of subjects tested positive for at least one respiratory virus, and $38.7 \%$ of these were infected with circulating Human Corona viruses (7). Rothe et al. described transmission of SARS-CoV-2 from a German patient who was infected by a Chinese businesswoman who visited Germany (5). Two other German co-workers were infected but only came in contact with the German patient who was asymptomatic. Mizumoto et al. (3) reported that $50.5 \%$ of infected patients on board the Diamond Princess cruise-ship were asymptomatic at time of diagnosis. Using a model, they found that the estimated asymptomatic proportion (among all infected cases) was $17.9 \%$ (95\%CI: 15.5-20.2\%). A study of evacuated Japanese nationals from Wuhan China, estimated that $30.8 \%$ of subjects were asymptomatic (95\% CI: $7.7 \%-53.8 \%)$ (8). A recent modeling study estimated that COVID-19 may be most transmissible in the two days prior to symptom onset (9). Another recent study of 3184 infections in Japan, identified 61 case-clusters, at various public events (10). Since both asymptomatic and pre-symptomatic subjects can spread the disease $(1,2)$, identifying such individuals is critical for effective control of the SARS-CoV-2 pandemic.

A major bottleneck of managing the COVID-19 pandemic in many countries is diagnostic testing which is primarily performed on symptomatic patients, due to limited laboratory capabilities as well as limited access to genome-extraction and Polymerase Chain Reaction (PCR) reagents. On the other hand, there is an urgent need to increase diagnostic testing capabilities in order to allow screening of asymptomatic and pre-symptomatic populations. In fact, such tests will be routinely required until a vaccine is developed.

Here, we developed P-BEST - a method for Pooling-Based Efficient SARS-CoV-2 Testing, using a single stage non-adaptive group-testing approach, which significantly reduces the number of tests required to identify all positive subjects within a large set of samples. P-BEST can be configured based on the carrier rate of a given population, and as we show below, if the carrier rate is below $1.3 \%$ the method provides both an 8-fold improvement in testing efficiency as well as an 8- 
fold reduction in test costs.

\section{Results \\ P-BEST overview}

To reduce the number of tests required for identifying all carriers, P-BEST uses a single step (non-adaptive) group testing design. Instead of testing each sample separately, samples are pooled into groups and each pool is tested for SARS-CoV-2 using a standard clinically approved PCR-based diagnostic assay. Each sample is part of multiple pools, using a combinatorial pooling strategy designed for maximizing the ability to identify all positive individuals $(11,12)$. PCR results for each of the pools are provided to the detection algorithm (see Methods), which identifies all positive carriers without the need for an additional testing stage (Fig. 1a-c). Importantly, pooling is performed on samples prior to RNA extraction. The efficiency of group testing (i.e., the ratio of the number of samples to the number of pools) is affected by the positive carrier rate of the population tested. As the carrier rate increases the efficiency is reduced, since more pools are required to correctly identify all positive subjects. If the percentage of carriers in the tested set of samples is $\sim 1 \%$, our method can correctly identify all positive individuals using an 8 -fold reduction in the number of diagnostic tests as compared to testing each individual sample separately.

\section{P-Best correctly identified all positive carriers in a set of 384 samples using only 48 pools}

We tested P-BEST using a pooling design constructed for carrier rates of up to $\sim 1 \%$ (i.e., 4 positive carriers within a set of 384 samples). We evaluated P-BEST using leftover samples that were previously clinically tested for COVID-19. 384 samples diluted in lysis buffer were pooled into 48 pools, each containing a set of 48 unique samples. Pooled samples were then tested by the clinical diagnostic laboratory of the Soroka University Medical Center (SUMC) using a clinically approved COVID-19 PCR-based diagnostic protocol that included an RNA extraction stage. We tested P-BEST using four sets of 384 samples, each containing an increasing number of positive carriers ranging from two to five. We found that PBEST was able to correctly identify all positive carriers within these four sets of 384 samples using only 48 tests per set, providing an 8-fold increase in testing efficiency (Fig. 1d). Only in the case of five carriers (carrier rate of 1.3\%) one additional false positive carrier was detected.

\section{PCR sensitivity ofpooled samples}

A key issue with sample pooling is loss of sensitivity due to sample dilution. In general, pooling a positive sample with additional negative samples will result in dilution of the viral RNA concentration within the pool. Theoretically, pooling 8 samples will result in a reduction of $\sim 3$ PCR cycles, and pooling 16 samples will lead to a reduction of $\sim 4$ PCR cycles, etc. Since in P-BEST samples are diluted into pools of 48 subjects, there is an inherent drop in PCR sensitivity of about 56 cycles (a factor of $2^{5}-2^{6}$ ), which was indeed observed experimentally. In retrospective analysis, we found that only a single pool that included a carrier yielded a negative PCR result (out of 70 positive pools across four experiments). Similarly, only $1 / 122$ of the negative pools was positively identified by the PCR-based assay. These two errors had no effect on the detection capabilities of P-BEST, which is robust to both of these types of errors.

To test the effect of pool size on PCR sensitivity, we conducted two independent experiments in which positive samples were diluted into sets of negative samples. In our first experiment a set of 5 positive samples (with $\mathrm{C}(\mathrm{t})$ values ranging from 26.2 to 34.2 ) were individually mixed into a set of 7 , 15, 19 and 23 negative samples generating pools of size 8, 16, 20 and 24 samples, respectively. Each single sample and pools were then tested for SARS-Cov-2 using an in-house PCR kit based on the E gene. We found that all pools were PCR positive, yet in some cases larger pool size actually yielded lower $\mathrm{C}(\mathrm{t})$ (Fig. 2a). In a second experiment another set of 5 samples (with $\mathrm{C}(\mathrm{t})$ values ranging from 25.2 to 30.8 ) were mixed with three distinct sets of 15 and 47 negative samples, resulting in pools of size 16 and 48, respectively. RNA was extracted from each single sample and pools and was then tested for SARS-CoV-2 using the Seegene COVID-19 diagnostic kit. We found that all 5 samples were positively detected in all of the pools of both size 16 and 48 (Fig. 2b). The level of variability within each triplicate differed by sample.

\section{In silico evaluation of P-BEST}

The performance of P-BEST was also evaluated using in silico simulations. In each simulation, a certain number of carriers (ranging from 1-5) were randomly selected within sets of 384 samples and a P-BEST experiment was simulated using the reported pooling design. Simulations included two sources of noise: (i)PCR failure which can lead to dropped pools; and (ii) Liquid dispensing volume errors corresponding to $50 \%$ error in the amount of RNA. In each such simulation, we quantified the number of false negatives, false positives and true positives returned by our method. Simulations demonstrated that the method can correctly identify up to $5 / 384$ (1.3\%) of carriers, with an average number of false positives that was less than 2.75 , and an average number of false negatives that was less than 0.33 (Fig. 3).

\section{P-BEST robustness}

To test the robustness of P-BEST we considered two types of potential noise factors. First, variation in initial RNA levels may cause samples to 'disappear' from all or part of the pools. Variation in RNA levels were estimated from Qubit 
measurements of 48 samples. The average RNA concentration was $15 \mathrm{ng} / \mu \mathrm{l}$, with standard deviation of $7 \mathrm{ng} / \mu \mathrm{l}$ (Supplemental Fig. 1A). These values were used in our simulations. A second possible source of noise is due to PCR amplification, which may fail in a certain number of pools. Figures 4-5 display the effects of these two noise factors on our decoding accuracy. The average number of false negatives and false positives did not change significantly.

\section{Using P-BEST to screen asymptomatic healthcare rork- ers}

We are conducting an ongoing clinical study to screen asymptomatic healthcare personnel of the SUMC medical center using P-BEST. Thus far, we approached 1,118 healthcare employees, of them 1,115 agreed to participate and signed an informed consent form. Within the cohort, 690 (62\%) were female and 425 (38\%) were male. Subjects were recruited across all SUMC staff and included: physicians $(n=165$, $14.8 \%)$, nurses $(n=157,14.1 \%)$, nurse assistants $(n=43,3.9 \%)$, other clinical staff $(n=119,10.7 \%)$ and administrative staff $(\mathrm{n}=631,56.6 \%) .296(26.5 \%)$ subjects worked in direct contact with COVID-19 patients. Within the cohort, 926 (93.1\%) subjects reported themselves as totally asymptomatic, 71 (6.3\%) reported a mild cough and 70 (6.3\%) reported rhinorrhea. The 1,115 participants were tested using three rounds of PBEST utilizing a total of $3 \times 48$ testing kits. All of the pools tested were negative. Due to increasingly lower carrier rates in Israel during April 2020, the third batch was blindly spiked with a sample from a COVID-19 patient. As in our preliminary validation studies, we correctly identified this patient using the P-BEST method.

\section{Discussion}

Here we described P-BEST - a novel method for efficient SARS-Cov-2 diagnostic testing that is based on single-stage non-adaptive group-testing. Using a pooling scheme designed for a carrier rate of $\sim 1 \%$ we showed that our method correctly identified all positive carriers in sets of 384 samples pooled into 48 pools, thereby providing an 8 -fold reduction in the number of required tests.

Any long-term mitigation strategy that will allow our society to exit from stringent lockdowns will require significant increases in testing capacity $(13,14)$. While it is clear that in many regions around the world the current carrier rates are significantly higher than $8 \%$, the lockdown periods imposed in many places are slowly reducing infection rates. Therefore, we anticipate that in the near future carrier rates are likely to drop below this threshold in many countries. P-BEST may then be used for conducting efficient routine screens of, e.g., health-care workers and staff in nursing homes, as well as general population screens to identify new hotspots of the SARS-CoV-2.
Recently several studies reported preliminary results using adaptive two-stage group-testing methods for SARS-CoV2 testing $(13,15,16)$. Additional reports have analyzed the efficiency gain of implementing pooled-testing (14, 17, 18). Furthermore, the FDA recently published novel guidelines on sample pooling (19). A clear advantage of P-BEST over other recently proposed pooling methods for SARS-CoV-2 diagnostic testing $(20,21)$ is that our method is a non-adaptive single stage method. Carriers are detected using a single round of testing, which reduces the overall test time, and does not require storing samples for a second round of testing. The latter is highly attractive for high throughput diagnostic labs for which logistical simplicity is imperative. Recently, another method for single stage COVID-19 group-testing was suggested (22).

Our current implementation of P-BEST was designed for a carrier rate of $\sim 1 \%$. To allow higher testing efficiency, i.e., to minimize the number of pools required to screen a given population, designs should vary according to the carrier rate. Pooling designs can differ by the number of pools, and by the number of samples per pool. Specifically, as the carrier rate in the population rises more pools are required to correctly identify all positive carriers in a single testing round. Moreover, the required number of samples per pool decreases with increasing the carrier rate. Conversely, when carrier rates are low, more efficient pooling designs can be used, i.e., less pools are required to test the same number of individuals, while the number of samples per pool should be increased. As the pandemic continues to spread, the infection rates within a given population are continuously changing. To provide a close to optimal pooling design a rough estimate of the current carrier rate is required. In general, this rate can be estimated based on the carrier rates observed during the last few days for a given population, since they typically gradually vary over time.

Each pooling design can correctly identify up to a certain percentage of carriers for which it was optimized. Therefore, carrier rates lower than the expected rate would not hamper detection (yet higher efficiency could have been achieved had this been known in advance). In contrast, the scenario in which the number of carriers is higher than expected may be problematic. As we show in our simulation results above, if the actual carrier rate ends up being slightly higher than the expected rate, our pooling method will identify all positive carriers, as well as some additional false positives, without the addition of false negatives. Such graceful degradation is required to handle variations in carrier rate that are either due to real changes, or result from the small sample size tested in each pooling run. Cases where the actual carrier rate is much higher than the expected level may also be identified using our method. In such case too many pools would be PCR positive, and the number of samples identified by the method 
will be much larger than the number of expected carriers. While this prevents the direct identification of the actual positive carriers using a single testing round, it will notify the user, and allow him/her to retest the samples either individually, or by using an alternate pooling design suited for a higher carrier rate.

As mentioned above, when carrier rates are sufficiently low, a significant increase in testing efficiency may be obtained, while requiring increasing the number of samples in each pool. Yet, increasing the pool size will lead to loss of sensitivity, and the optimal pool size should be quantified for each diagnostic kit. While two recent reports tested pooling sensitivity with pools of up to 32 samples per pool $(13,23)$, we found that samples with $\mathrm{C}(\mathrm{t})<35$ were detected in pools of up to 48 samples each, using two different SARS-CoV-2 diagnostic tests. The loss of sensitivity differed from sample to sample, and generally adhered to the theoretically expected value, however this was not always the case. We note that PBEST can be used with any pool size, yet limiting the pool size for low carrier rates will reduce its efficiency. For example, screening 384 samples using pools of size 16 (instead of 48 ) will require 72 pools, thereby providing a $\sim 5.3$-fold increase in testing efficiency (as compared to an 8 -fold increase with pools of 48).

We tested the performance of P-BEST in a clinical study aimed at screening asymptomatic and mildly symptomatic healthcare workers. Using P-BEST we were able to screen 1115 patients using a total of 144 clinical diagnostic kits, highlighting the efficiency of our method. Due to stringent lockdowns imposed in Israel, infection rates in April 2020 significantly dropped and the number of new cases per day continued to decline. We were therefore not surprised that no positive carrier was identified within the 1115 subjects screened thus far. The correct identification of a blindly spiked positive sample was used to verify that the pooling, RNA extraction and PCR processes were properly performed. We note that this would be one typical scenario where the use of P-BEST would be most suitable, i.e., carrier screening when infection rates are very low $(<1 \%)$, thus providing significant savings in reagents and other diagnostic testing resources, as well as significantly increasing testing capacity.

We are currently exploring several improvements of our method as follows: (i) P-BEST requires the use of widely available automated liquid dispensing robots. While our current application of P-BEST required $\sim 5$ hours for pool assembly using a single channel robot, we have now successfully tested a more advanced liquid handling system with a multi-dispenser arm that can perform this task in about 1 hour. Importantly, our method can be easily adapted to work on any standard liquid dispensing robot, and we are in the process of developing code for such robots from several leading vendors; (ii) to allow P-BEST to be entirely performed in a BSL-
2 laboratory we are currently experimenting with two inactivation methods: directly depositing swab samples into tubes with lysis buffer instead of viral transfer media, or heating samples at $70^{\circ} \mathrm{C}$ for $30 \mathrm{~min}$; (iii) Compressed sensing detection in P-BEST can, in principle, estimate the viral load $(\mathrm{C}(\mathrm{t})$ values) of each positive carrier. Such estimates may prove clinically relevant and are straightforward in P-BEST; (iv) Due to the global shortage of RNA extraction kits, we are currently testing heat-based and detergent-based methods RNA extraction protocols (24). While these protocols will be performed in a BSL-3 laboratory, they will reduce the test time by about 2 hours, and will also reduce test costs by about $50 \%$; and (v) A recent report has suggested that saliva is more sensitive than nasopharyngeal swabs for SARS-CoV-2 detection (25). Using saliva samples, which are self-collected, can greatly increase sample collection capacity. We are, therefore, also optimizing our method for pooling saliva samples.

In summary, here we presented a novel method for efficient SARS-CoV-2 diagnostic testing that can provide significant increases in both testing efficiency and testing resources, and can be readily implemented in clinical diagnostic labs. Code and protocols required for implementation of P-BEST can be found at https://github.com/NoamShental/PBEST.

\section{Methods \\ Group testing and sample pooling method}

The mathematical field of group testing (26) aims to tackle the problem of efficiently identifying individuals carrying a certain rare trait within a large population by pooling samples and testing each pool as if it were a single sample. In general, pooling is designed in such a way that each individual has a unique 'footprint' within the set of pools, thus allowing carrier identification. Group testing dates back to the mid-20th century (27), and since then many intricate pooling designs have been described and rigorously analyzed. Group testing has been successfully applied in data compression (28), computation in the data stream model (29) and in molecular biology $(30,31)$. In our previous studies we described a combination of group testing and next generation sequencing for detecting carriers of rare genetic mutations. We detected all individual carriers of rare mutations out of a set of 1024 mutagenized Sorghum bicolor plants using a set of 48 pools $(11,12)$.

The efficiency of group testing, often measured by the ratio between the number of screened individuals to the number of pools, generally increases with the decreasing frequency of the observed trait. For example, screening for a trait that appears in $0.1 \%$ of the population can be done more efficiently than screening for a trait that appears in $1 \%$ of the population. Moreover, when the carrier rate exceeds $\sim 8 \%$ group testing is no longer effective, since the required number of pools would be comparable to the number of samples 
tested.

To optimize efficiency, the pooling design needs to be tailored to the expected carrier rate, by adjusting the number pools and the number of samples per pool. If the true carrier rate exceeds the expected rate, the method will identify larger sets of suspected carriers, which may include false positives, without any additional false negatives. Therefore, it is imperative to evaluate the robustness of a specific pooling design to higher carrier rates (e.g., Fig. 2). In case the true carrier rate is much lower than the rate for which the pooling design was tailored, testing efficiency would be suboptimal, since a smaller number of pools would have sufficed to correctly detect all positive carriers.

\section{P-BEST pooling design}

In our current proof-of-concept study of P-BEST, we developed a pooling scheme designed to correctly identify all positive carriers for carrier rates $<1.3 \%$. Specifically, we pooled sets of 384 patient samples into 48 pools, each containing 48 samples. Each sample was added to six different pools. Pools were designed based on a Reed-Solomon error correcting code (32) which as in our previous work, proved to be robust to experimental noise, e.g., pools that fail to be amplified.

\section{P-BEST detection algorithm}

PCR samples with $\mathrm{C}(\mathrm{t})$ values $<40$ were considered positive. Carrier detection was performed using the Gradient Projection for Sparse Reconstruction (GPSR) algorithm (33) as in our former studies $(11,12)$. The transformation from fractional to discrete results was done using the following algorithm: The 20 samples with highest scores were selected and only subsets of these 20 were further considered. In total $2^{20}$ subsets of samples were tested. Each subset corresponds to a vector $\mathbf{x}$ of length 384, in which the entries of the selected samples were equal to 1 , and all others were set to zero. Nonzero entries of the product of $M x$, where $M$ is the pooling matrix, were replaced by the value 1 , and compared to the (binary) measurement vector $\mathbf{y}$. The vector $\mathbf{x}^{*}$ for which $\left\|M \mathbf{x}^{*}-y\right\|_{1}$ achieved its minimum was selected.

\section{P-BEST in silico simulations}

In each simulation, a number of carriers (ranging from 1-5) were randomly selected, thus corresponding to a vector $\mathbf{x}$ of length 384 , in which the selected carriers were equal to 1 , and all others were set to zero. The measurement vector $\mathbf{y}$ representing the PCR measurements over the P-BEST pools was provided by binarizing $M^{\prime} \mathbf{x}$, where $M^{\prime}$ is a noisy instance of the pooling matrix $M$. More specifically, to simulate liquid dispensing volume errors, each nonzero entry in $M$ was sampled from a normal distribution with a mean of 1 and standard deviation of 0.01. A second type of experimental noise, corresponding to dropped pools due to PCR failure, was simulated by randomly deleting entries in $\mathbf{y}$ (and corresponding rows in $M$ ). The detection algorithm presented in the former section was used to identify carriers using the vector $\mathbf{y}$ and the matrix $M$ as input, and its detected carriers were compared to $\mathbf{x}$ to evaluate performance.

\section{SARS Cov-2 clinical diagnostics}

Naso and oropharynx swabs were collected for analysis by the laboratory of Clinical Virology in Soroka University Medical Center (SUMC), which is approved by the Israeli Ministry of Health to test for SARS-CoV-2 infections. The laboratory uses a clinically approved 2019-nCoV detection kit (Seegene, California, USA) for both viral nucleic acid extraction and qRTPCR-based amplification. The kit identifies three SARS-CoV2 genes: E, RdRP and N genes. RNA extraction was performed using the STARMag 2019-nCoV kit (Seegene, California USA) on a liquid dispensing robot (STARlet Hamilton, USA). All samples were analyzed individually, and positive and negative results were recorded by the SUMC diagnostic lab, prior to our study. To inactivate the virus and extract viral RNA, $500 \mathrm{\mu l}$ of the transfer medium containing the swab are combined with $350 \mu \mathrm{l}$ of lysis buffer of the STARMag kit in a BSL$3 \mathrm{lab}$; following 20' of incubation, $350 \mu \mathrm{l}$ were used for nucleic acid extraction into a volume of $100 \mu$ l extracted genome, from which $8 \mu \mathrm{l}$ were taken for the 2019-nCoV PCR assay.

\section{Clinical cohorts}

For initial validation of P-BEST, we used leftover clinical samples from patients who tested positive and patients who tested negative from the clinical virology lab of SUMC. Four sets of 384 samples each containing 2-5 positive samples (Sets $1-4$, respectively) were created. We then conducted a clinical study to screen SUMC healthcare workers for SASR-CoV-2 infections. The study recruited adult subjects that self-reported as asymptomatic or mildly symptomatic (cough, runny nose, myalgia or sore throat) with no fever, shortness of breath or pneumonia. After signing an informed consent, samples were obtained by swabbing the nasopharynx and oropharyngeal cavity each with a different swab using standard techniques. Three batches of 384 participants were collected. As a positive control, one collection batch included a sample from a patient who tested positive via an individual PCR-based diagnostic method. This study was approved by SUMC ethical review board (Helsinki committee).

\section{P-BEST experimental setup}

Pools were prepared using a liquid handling robot (Arise Ezmate-601) using a code written in Python. The code automatically generates a command file for the robot to use. Samples were manually pipetted into 96 well plates from which the robot assembled a set of 48 pools, each containing 48 distinct 
samples. We generated 48 pools for each sample set. Each pool contained equal volumes from 48 samples (11 $\mu \mathrm{l}$ per sample, $528 \mu$ l per pool). Each individual sample was represented in six different pools. The total time for pooling 384 samples into 48 pools was $\sim 5$ hours, and was performed in a standard BSL-2 laboratory. Analogously to single samples, $350 \mu \mathrm{l}$ from each pool were then used for nucleic acid extraction into a volume of $100 \mu \mathrm{l}$, using the clinically approved STARMag kit and the STARlet robot (Hamilton, USA). Then, $8 \mu$ l were used for qRT-PCR to detect the E gene of SARS-CoV-2, based on an in-house method that was clinically validated and employed by the SUMC-clinical virology laboratory prior to the introduction of the 2019-nCoV Seegene kit. We used this PCR method due to shortages of the Seegene 2019-nCoV assay kits used by the clinical diagnostic lab at SUMC. To verify compatibility of the Seegene kit, we re-tested some of our positive pools with the Seegene PCR kit. Pools for the clinical study were generated as above, but $21 \mu l$ per sample were used to obtain a total volume of $1008 \mu \mathrm{l}$ in each pool. Testing of the samples collected in the clinical study was performed using the clinically approved Seegene assay kits.

\section{REFERENCES AND NOTES}

1. Y. Bai, L. Yao, T. Wei, F. Tian, D.-Y. Jin, L. Chen, M. Wang, Presumed Asymptomatic Carrier Transmission of COVID-19. JAMA 323, 1406 (2020). doi:10.1001/iama.2020.2565 Medline

2. R. Li, S. Pei, B. Chen, Y. Song, T. Zhang, W. Yang, J. Shaman, Substantial undocumented infection facilitates the rapid dissemination of novel coronavirus (SARS-CoV-2). Science 368, 489-493 (2020). doi:10.1126/science.abb3221 Medline

3. K. Mizumoto, K. Kagaya, A. Zarebski, G. Chowell, Estimating the asymptomatic proportion of coronavirus disease 2019 (COVID-19) cases on board the Diamond Princess cruise ship, Yokohama, Japan, 2020. Euro Surveill. 25, (2020). doi:10.2807/1560-7917.ES.2020.25.10.2000180 Medline

4. L. F. Moriarty, M. M. Plucinski, B. J. Marston, E. V. Kurbatova, B. Knust, E. L. Murray, N. Pesik, D. Rose, D. Fitter, M. Kobayashi, M. Toda, P. T. Cantey, T. Scheuer, E. S. Halsey, N. J. Cohen, L. Stockman, D. A. Wadford, A. M. Medley, G. Green, J. J. Regan, K. Tardivel, S. White, C. Brown, C. Morales, C. Yen, B. Wittry, A. Freeland, S. Naramore, R. T. Novak, D. Daigle, M. Weinberg, A. Acosta, C. Herzig, B. K. Kapella, K. R. Jacobson, K. Lamba, A. Ishizumi, J. Sarisky, E. Svendsen, T. Blocher, C. Wu, J. Charles, R. Wagner, A. Stewart, P. S. Mead, E. Kurylo, S. Campbell, R. Murray, P. Weidle, M. Cetron, C. R. Friedman, CDC Cruise Ship Response Team; California Department of Public Health COVID-19 Team; Solano County COVID-19 Team, Public Health Responses to COVID-19 Outbreaks on Cruise Ships Worldwide, February-March 2020. MMWR Morb. Mortal. Wkly. Rep. 69, 347-352 (2020). doi:10.15585/mmwr.mm6912e3 Medline

5. C. Rothe, M. Schunk, P. Sothmann, G. Bretzel, G. Froeschl, C. Wallrauch, T. Zimmer, V. Thiel, C. Janke, W. Guggemos, M. Seilmaier, C. Drosten, P. Vollmar, K. Zwirglmaier, S. Zange, R. Wölfel, M. Hoelscher, Transmission of 2019-nCoV Infection from an Asymptomatic Contact in Germany. N. Engl. J. Med. 382, 970971 (2020). doi:10.1056/NEJMc2001468 Medline

6. L. Zou, F. Ruan, M. Huang, L. Liang, H. Huang, Z. Hong, J. Yu, M. Kang, Y. Song, J. Xia, Q. Guo, T. Song, J. He, H.-L. Yen, M. Peiris, J. Wu, SARS-CoV-2 Viral Load in Upper Respiratory Specimens of Infected Patients. N. Engl. J. Med. 382, 11771179 (2020). doi:10.1056/NEJMc2001737 Medline

7. R. Birger, H. Morita, D. Comito, I. Filip, M. Galanti, B. Lane, C. Ligon, D. Rosenbloom, A. Shittu, M. Ud-Dean, R. Desalle, P. Planet, J. Shaman, Asymptomatic Shedding of Respiratory Virus among an Ambulatory Population across Seasons. MSphere 3, e00249-18 (2018). doi:10.1128/mSphere.00249-18 Medline

8. H. Nishiura, T. Kobayashi, T. Miyama, A. Suzuki, S. M. Jung, K. Hayashi, R. Kinoshita,
Y. Yang, B. Yuan, A. R. Akhmetzhanov, N. M. Linton, Estimation of the asymptomatic ratio of novel coronavirus infections (COVID-19). Int. J. Infect. Dis. 94, 154-155 (2020). doi:10.1016/i.iiid.2020.03.020 Medline

9. X. He, E. H. Y. Lau, P. Wu, X. Deng, J. Wang, X. Hao, Y. C. Lau, J. Y. Wong, Y. Guan, X. Tan, X. Mo, Y. Chen, B. Liao, W. Chen, F. Hu, Q. Zhang, M. Zhong, Y. Wu, L. Zhao, F. Zhang, B. J. Cowling, F. Li, G. M. Leung, Temporal dynamics in viral shedding and transmissibility of COVID-19. Nat. Med. 26, 672-675 (2020). doi:10.1038/s41591020-0869-5

10. Y. Furuse et al., Clusters of Coronavirus Disease in Communities, Japan, JanuaryApril 2020. .Emerg.Infect. Dis. 26,10.3201/eid2609.202272 (2020).

11. N. Shental, A. Amir, O. Zuk, Identification of rare alleles and their carriers using compressed se(que)nsing. Nucleic Acids Res. 38, el79 (2010). doi:10.1093/nar/gkq675 Medline

12. H. Nida, S. Blum, D. Zielinski, D. A. Srivastava, R. Elbaum, Z. Xin, Y. Erlich, E. Fridman, N. Shental, Highly efficient de novo mutant identification in a Sorghum bicolor TILLING population using the ComSeq approach. Plant J. 86, 349-359 (2016). doi:10.1111/tpj.13161 Medline

13. S. Lohse, T. Pfuhl, B. Berkó-Göttel, J. Rissland, T. Geißler, B. Gärtner, S. L. Becker, S. Schneitler, S. Smola, Pooling of samples for testing for SARS-CoV-2 in asymptomatic people. Lancet Infect. Dis. S1473-3099(20)30362-5 (2020). doi:10.1016/S1473-3099(20)30362-5 Medline

14. Majid, F., S.B. Omer, and A.I. Khwaja, Optimising SARS-CoV-2 pooled testing for low-resource settings. Lancet Microbe 1, e101-e102 (2020).

15. C. A. Hogan, M. K. Sahoo, B. A. Pinsky, Sample Pooling as a Strategy to Detect Community Transmission of SARS-CoV-2. JAMA 323, 1967-1969 (2020). doi:10.1001/jama.2020.5445 Medline

16. R. Ben-Ami et al., Large-scale implementation of pooled RNA-extraction and RTPCR for SARS-CoV-2 detection. medRxiv, 2020: p. 2020.04.17.20069062.

17. B. Abdalhamid, C. R. Bilder, E. L. McCutchen, S. H. Hinrichs, S. A. Koepsell, P. C. Iwen, Assessment of Specimen Pooling to Conserve SARS CoV-2 Testing Resources. Am. J. Clin. Pathol. 153, 715-718 (2020). doi:10.1093/aicp/aqaa064 Medline

18. N. Sinnott-Armstrong, D. Klein, B. Hickey, Evaluation of Group Testing for SARSCoV-2 RNA. medRxiv, 2020: p. 2020.03.27.20043968.

19. J. E. Shuren, Coronavirus (COVID-19) Update: Facilitating Diagnostic Test Availability for Asymptomatic Testing and Sample Pooling. [July 12, 2020]; Available from: https://www.fda.gov/news-events/pressannouncements/coronavirus-covid-19-update-facilitating-diagnostic-testavailability-asymptomatic-testing-and

20. I. Yelin et al., Evaluation of COVID-19RT-qPCR test in multi-sample pools. medRxiv, 2020: p. 2020.03.26.20039438.

21. R. Ben-Ami et al., Pooled RNA extraction and PCR assay for efficient SARS-CoV-2 detection. medRxiv, 2020: p. 2020.04.17.20069062.

22. S. Ghosh et al., Tapestry: A Single-Round Smart Pooling Technique for COVID-19 Testing. medRxiv, 2020: p. 2020.04.23.20077727.

23. I. Yelin, N. Aharony, E. Shaer Tamar, A. Argoetti, E. Messer, D. Berenbaum, E. Shafran, A. Kuzli, N. Gandali, O. Shkedi, T. Hashimshony, Y. Mandel-Gutfreund, M. Halberthal, Y. Geffen, M. Szwarcwort-Cohen, R. Kishony, Evaluation of COVID-19 RT-qPCR test in multi-sample pools. Clin. Infect. Dis. ciaa531 (2020). doi:10.1093/cid/ciaa531 Medline

24. I. Smyrlaki et al., Massive and rapid COVID-19 testing is feasible by extraction-free SARS-CoV-2 RT-qPCR. medRxiv, 2020: p. 2020.04.17.20067348.

25. A. L. Wyllie et al., Saliva is more sensitive for SARS-CoV-2 detection in COVID-19 patients than nasopharyngeal swabs. medRxiv, 2020: p. 2020.04.16.20067835.

26. D. Z. Du, F. K. Hwang, Combinatorial Group Testing and Its Applications. Series on Applied Mathematics. Vol. Volume 3. 1993: WORLD SCIENTIFIC. 264.

27. R. Dorfman, The Detection of Defective Members of Large Populations. Ann. Math. Stat. 14, 436-440 (1943). doi:10.1214/aoms/1177731363

28. W. H. Kautz, R. C. Singleton, Nonrandom binary superimposed codes. IEEE Trans. Inf. Theory 10, 363-377 (1964). doi:10.1109/TIT.1964.1053689

29. G. Cormode, S. Muthukrishnan, What's hot and what's not: Tracking most frequent items dynamically. ACM Trans. Database Syst. 30, 249-278 (2005). doi:10.1145/1061318.1061325

30. S. Prabhu, I. Pe'er, Overlapping pools for high-throughput targeted resequencing. Genome Res. 19, 1254-1261 (2009). doi:10.1101/gr.088559.108 Medline 
31. Y. Erlich, K. Chang, A. Gordon, R. Ronen, O. Navon, M. Rooks, G. J. Hannon, DNA Sudoku-Harnessing high-throughput sequencing for multiplexed specimen analysis. Genome Res. 19, 1243-1253 (2009). doi:10.1101/gr.092957.109 Medline

32. I. S. Reed, G. Solomon, Polynomial Codes Over Certain Finite Fields. J. Soc. Ind. Appl. Math. 8, 300-304 (1959). doi:10.1137/0108018

33. M. A. Figueiredo, R. D. Nowak, S. J. Wright, Gradient projection for sparse reconstruction: Application to compressed sensing and other inverse problems. IEEE J. Sel. Top. Signal Process. 1, 586-597 (2007). doi:10.1109/JSTSP.2007.910281

\section{ACKNOWLEDGMENTS}

We thank Jenny Racah and Shelly Levy-Tzedek for constructive discussions and manuscript editing. Noam Shental would like to thank Dalia Shental and Menashe Sobel for useful discussions. Funding: Funding for this study was provided by a donation to BGU from the Rosetrees Trust. A.P. was supported by an Israeli Science Foundation grant ISF-2484/19 (10/2019-9/2023). T.H. was supported by an Israeli Science Foundation grant ISF-882/17 (10/2017-9/2021). Author contributions: N.S., T.H., and A.P. designed the study and supervised all experiments. Y.S.-A. supervised clinical testing and designed the experiments. L.N. conducted the clinical study. S.L., S.S., V.W., B.S., S.Sa., A.O., Y.G., A.E., and R.S. performed the experiments. R.G., M.G., K.M., S.L., and S.S. collected clinical samples. N.S., A.P., T.H., L.N., Y.S.-A., and L.M.F. wrote the paper. Competing interests: N.S., T.H., and A.P. are authors on a pending patent application related to this work filed by the National Institute of Biotechnology of the Negev and the Open University of Israel (no. 06953-P0029A, filed 13 April 2020). The other authors declare that they have no competing interests. Data and materials availability: All data needed to evaluate the conclusions in the paper are present in the paper, the Supplementary Materials, and/or the P-BEST Github page.

\section{SUPPLEMENTARY MATERIALS}

advances.sciencemag.org/cgi/content/full/sciadv.abc5961/DC1

Submitted 3 May 2020

Accepted 28 July 2020

Published First Release 21 August 2020

10.1126/sciadv.abc5961 


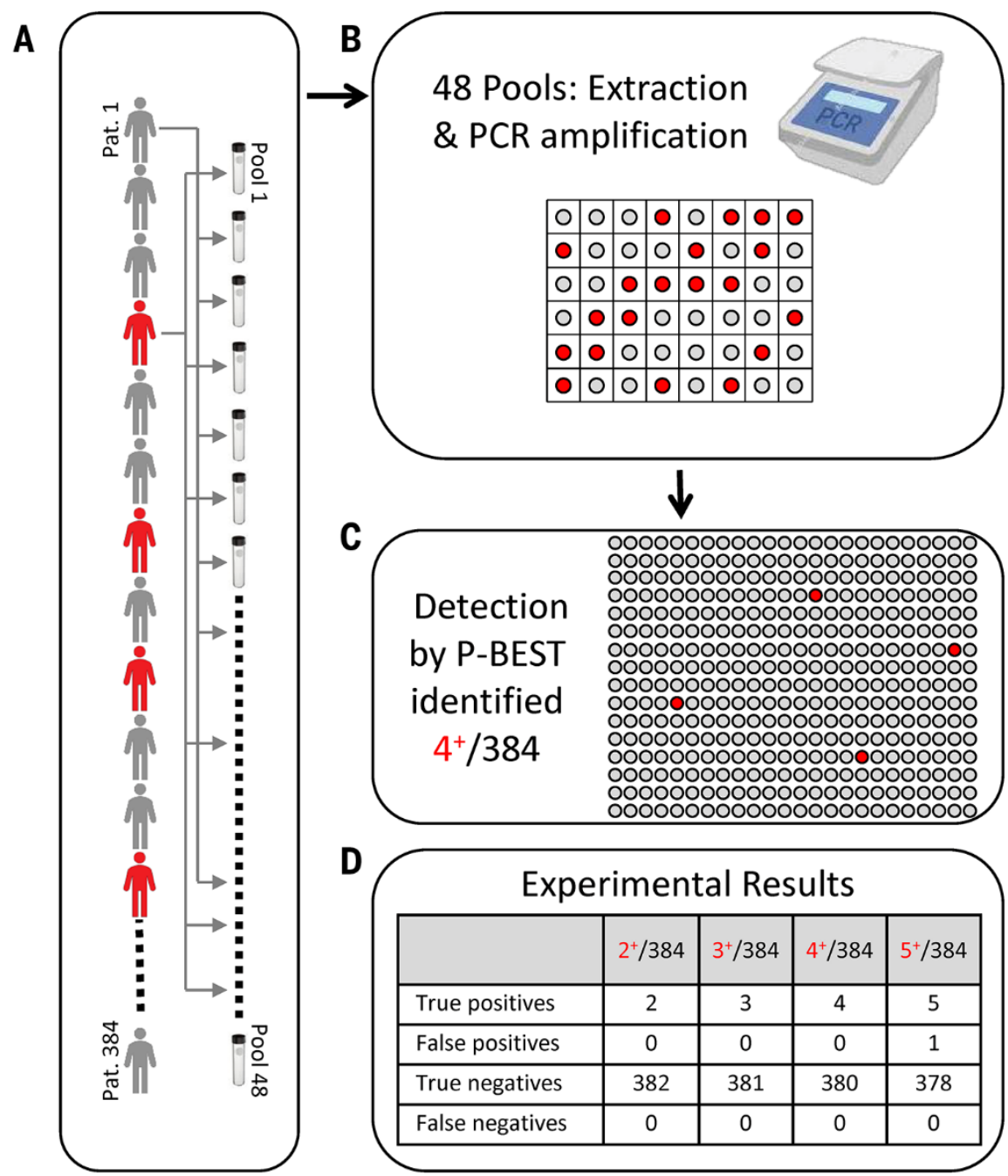

Fig. 1. P-BEST design and detection results obtained for a set of 384 samples with a carrier rate of $\sim 1 \%$. a. Pooling design - Pools are generated using a combinatorial pooling design based on an error correcting code that optimizes carrier detection. The P-BEST pooling design for a carrier rate of $\sim 1 \%$ uses 48 pools to test 384 subjects simultaneously providing both an 8-fold increase in testing efficiency, as well as an 8-fold reduction in testing reagent costs. Each sample is distributed to six pools and there are a total of 48 subjects per pool. Subjects in red represent the four unidentified infected individuals within the set of 384 samples. b. Pooled samples are then treated as individual samples - RNA is extracted, followed by standard PCR amplification. Positive pools are designated by red circles. c. PBEST identifies the positive samples out of the 384 samples using an optimization-based algorithm based on compressed sensing. d. Results of the four experiments performed, containing 2, 3, 4 or 5 positive samples, respectively. All positive carriers were correctly identified in all experiments. When five positive carriers were tested (carrier rate of $1.3 \%$ ), a single false positive sample was added to the true positive ones. 
A

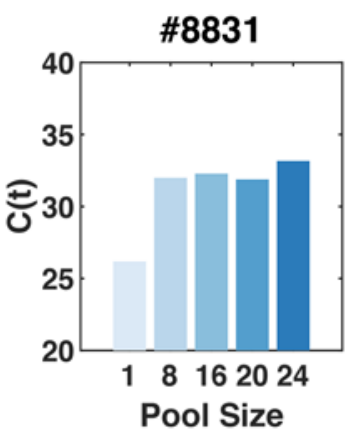

B

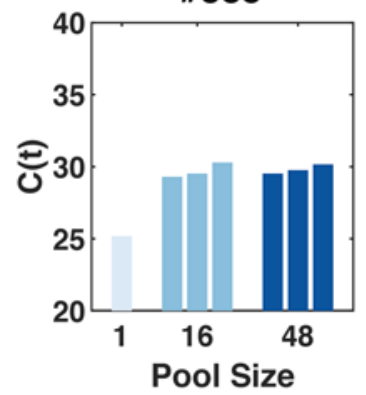

\#8922

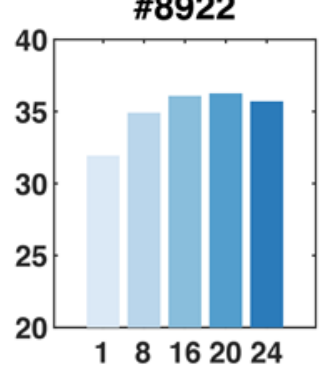

\#223

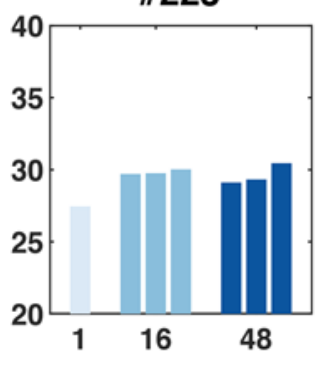

\#8923

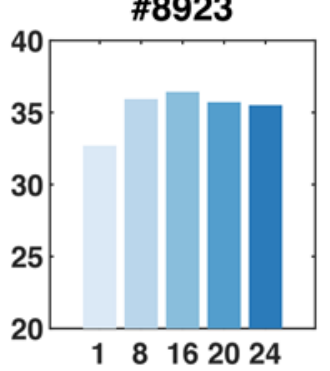

\#60

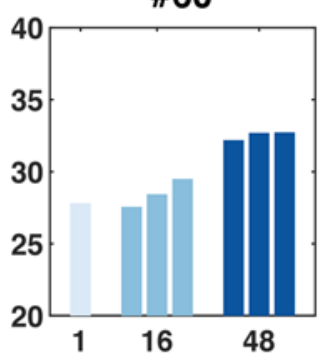

\#8937

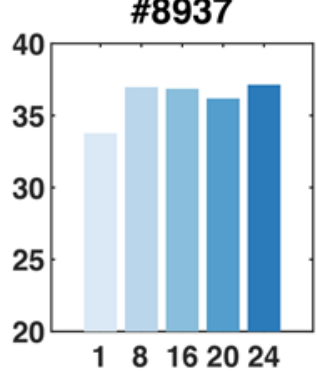

\#159

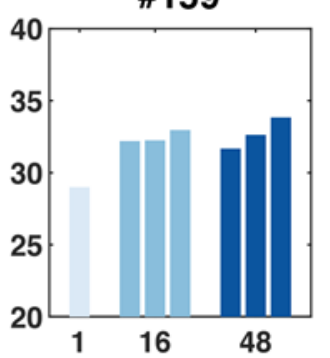

\#8933

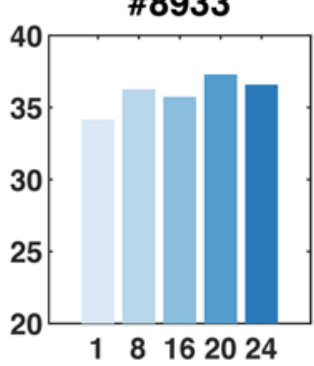

\#31

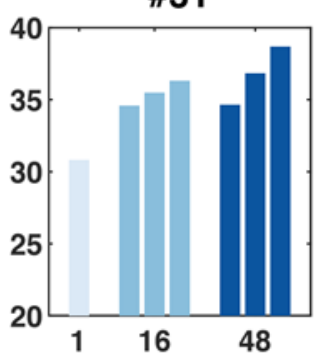

Fig. 2. The effect of sample dilution on $\mathrm{C}(\mathrm{t}$ ) values. (a) Five positive samples from the Soroka virology lab were heat-inactivated $\left(70^{\circ} \mathrm{C}, 60 \mathrm{~min}\right)$ and tested as individual samples and in pools of sizes $8,16,20$ and 24. For each pool size, the set of negative samples used were identical across all five samples tested, e.g., the same 7 negative samples were used to create all pools of size 8. Sample's de-identified study IDs appear above each subplot. Each pool size is plotted in a different color. All samples, including those with $C(t)>34$ were identified in all pool sizes, yet in some cases the $C(t)$ value does not monotonically increase with pool size. Samples were tested using an in-house PCR kit based on the SARS-CoV-2 E gene. (b) Five positive samples were mixed into 3 pools of sizes 16 and 48, each containing a distinct set of negative samples. Sample de-identified study IDs appear above each subplot. RNA was extracted from all single samples and pools and subsequently tested for SARS-CoV-2 using the Seegene diagnostic kit. All five samples were identified across all pools of both sizes, yet triplicates sometime display variation in $C(t)$. 
A

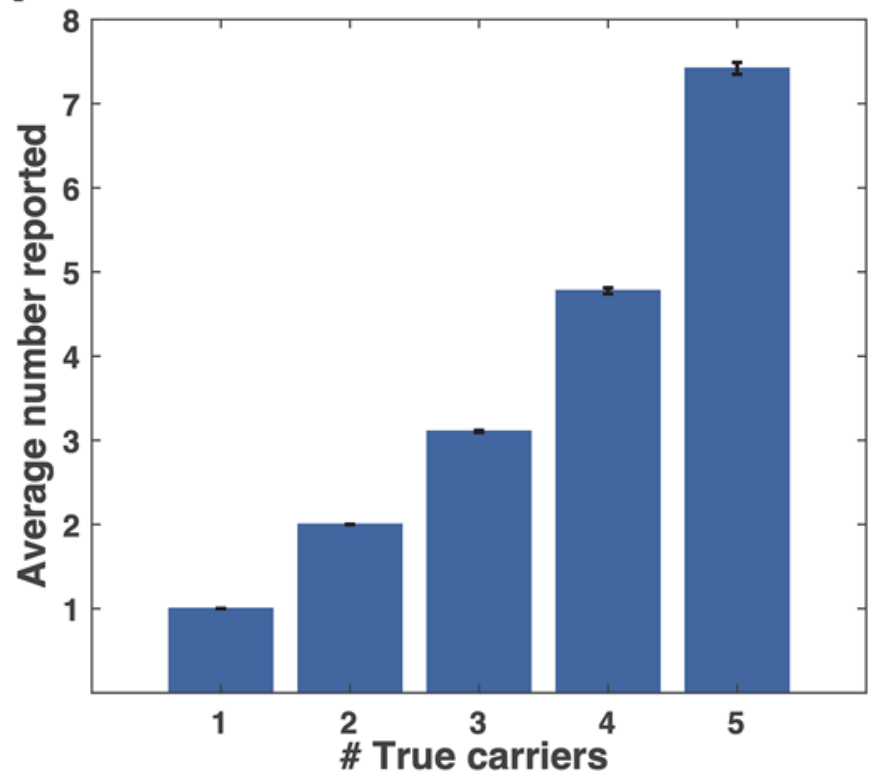

B

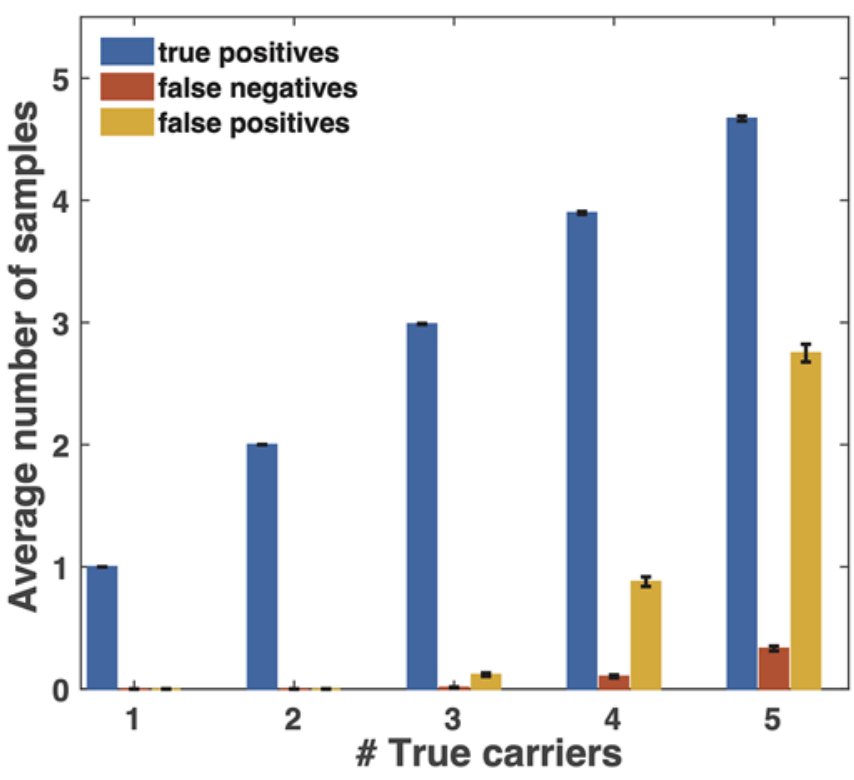

Fig. 3. P-BEST in silico performance. A small number of carriers were randomly assigned to 384 samples and a P-BEST experiment was simulated using the reported pooling scheme designed for a carrier rate of $\sim 1 \%$. Simulations accounted for the estimated variation in RNA amounts based on measurements of $\mathrm{n}=48$ individual samples, and also assumed that one of the 48 pools failed PCR amplification. Samples reported by P-BEST were compared to the true simulated sample labels to estimate P-BEST's success rate. Results were averaged over 3000 simulations. Error bars correspond to 95\% confidence intervals. (A) The average number of samples reported by P-BEST as a function of the number of true carriers. For example, P-BEST reports exactly two samples when simulating two carriers, and retrieves an average of 7.4 samples when the simulated set contains five carriers. (B) The average number of true positives, false negatives and false positives identified for a given number of simulated carriers. Even for five carriers the number of false negatives is lower than one, and the average number of false positives remains low $(<3)$. 

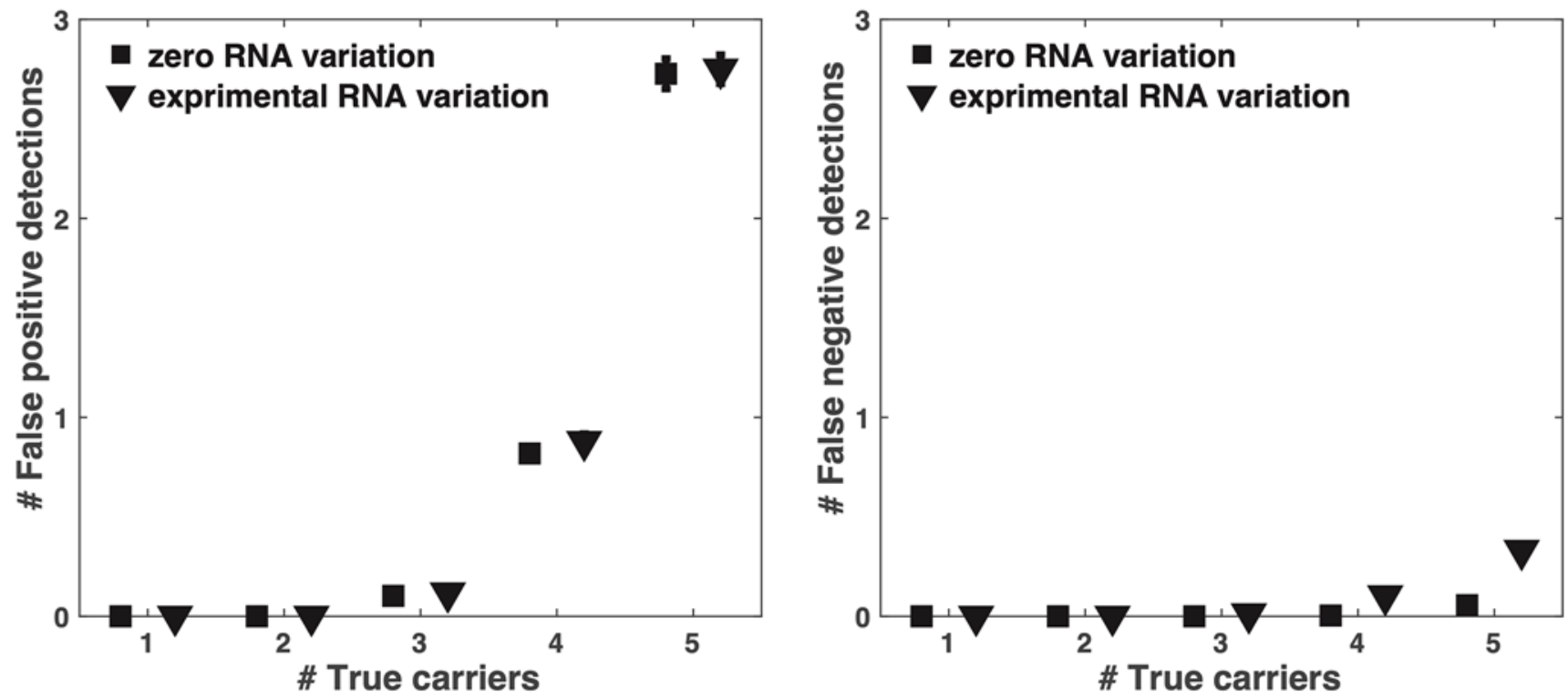

Fig. 4. Evaluating the effect of variation in RNA levels on P-BEST performance. To assess the effects of variations in RNA levels, we measured the average number of false positive and false negative detections as a function of the true number of carriers across 3000 simulations in two scenarios: (1) No noise in RNA levels (black square) and (2) RNA noise based on the measured variation of RNA levels across 48 samples (see Supplemental Fig. 1). Simulations used the pooling scheme designed for a carrier rate of $\sim 1 \%$. The false positive (left panel) and false negative (right panel) detection rates for the two scenarios show that RNA variation does not significantly degrade P-BEST performance. All simulations considered one dropped pool. Error bars correspond to 95\% confidence intervals. 

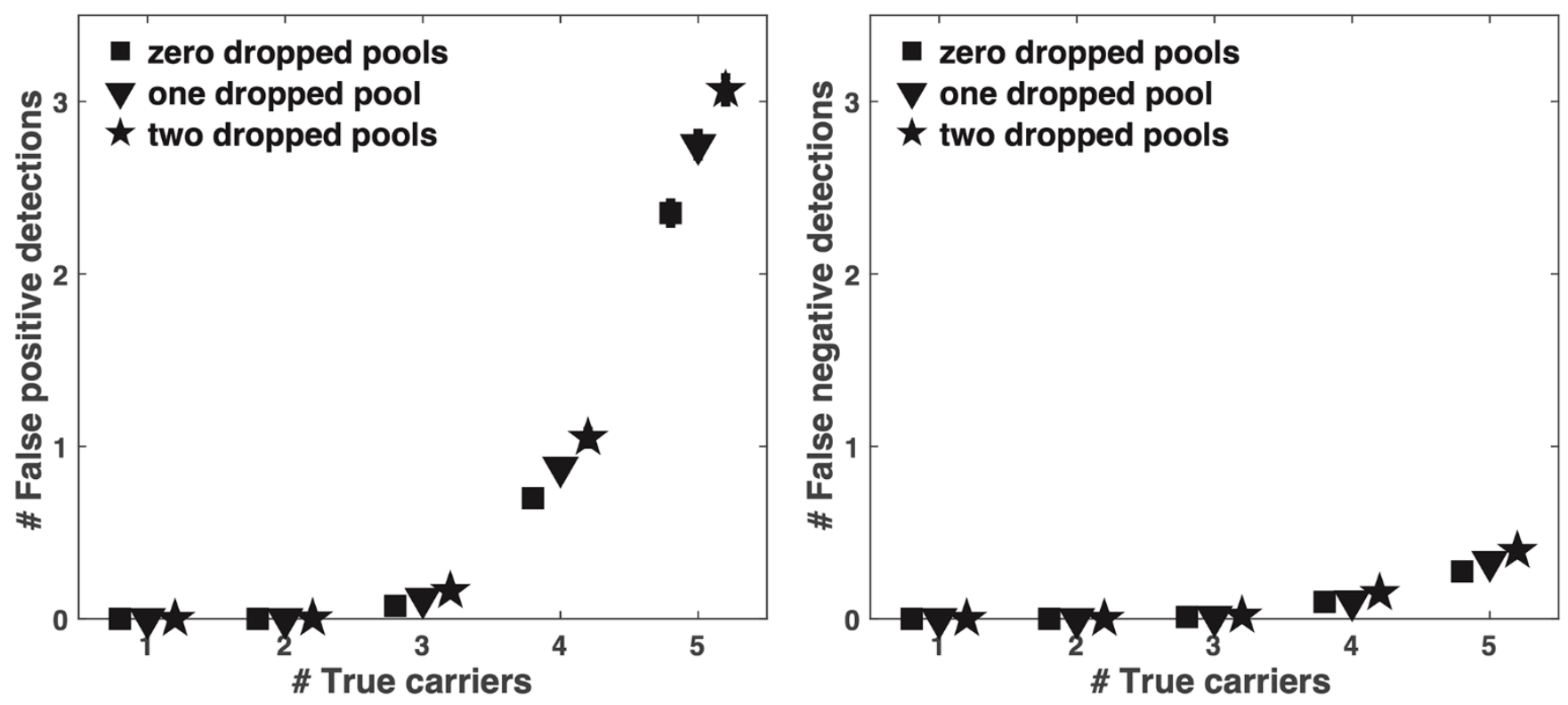

Fig. 5. Evaluating the effect of dropped pools on P-BEST performance.To assess the effects of dropped pools due to PCR amplification failures, we measured the average number of false positive (left panel) and false negative (right panel) detections as a function of the true number of carriers across 3000 simulations for zero, one or two randomly dropped pools using the pooling scheme designed for a carrier rate of $\sim 1 \%$. P-BEST seems to be robust to 1-2 dropped pools. All simulations considered the experimental level of RNA variation as measured across 48 samples (Supplemental Fig. 1A). Error bars correspond to 95\% confidence intervals. 